\title{
A study to evaluate predictors of mortality in mechanically ventilated patients using APACHE II and SAPS II

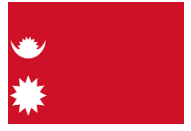

Raj Kumar Mehta RN; MSN (Critical Care), BSc (Hons) Nursing, Lecturer, Koshi Health \& Science Campus, Biratnagar, Nepal. Nursing Director, MediEscort School of Health Science, Dulari, Morang, Nepal.

Ashia Qureshi RN; MSN, Lecturer, College of Nursing, All India Institute of Medical Sciences, New Delhi, India

MK Arora MD, Professor, Dept. of Anesthesiology \& Critical Care, All India Institute of Medical Sciences, New Delhi, India

Key Words: APACHE II critically ill * illness severity scoring system * outcome * SAPS II *

E-mail: mehtarajkraj@gmail.com

\section{SUMMARY}

- Critical care is the care of seriously ill patients with life threatening illness or trauma; these patients also have the potential to develop life-threatening complications from their disease. Critical care should be reserved for patients with severe but potentially reversible problems.

- Critical care has grown into a specialty with special training and certification in the west, and also in many developed or quickly developing Asian countries. The specialty is relatively new but growing in developing countries like India. The chief advantage of the critical care unit is that it provides better and more organized care (Udwadia, 1995).

- The beds in the ICU are limited and cost of care is extremely high due to advanced technology and specialized professional care. Estimates of prognosis faced by the patient could be useful in identifying patients who are likely to survive with intensive care and treatment so that patients with an extremely poor prognosis do not occupy ICU beds (Schreiderman et al. 1990).

- Clinical research has focused on the ability to objectively predict a patient's risk for mortality. However, attempts at prediction have been successful in forecasting individual patient risk or in reducing the uncertainty of making daily clinical decision. It is well known that a large amount of ICU expenses can be attributed to patients with a poor outcome. This has stimulated an interest in identifying this segment of the ICU population early and possibly transitioning their care. Many methods have been developed for this purpose and fall under the headings of predictive instruments, illness severity indexes and mortality scoring system (Meyer \& Hunt, 1997).

- In this study, we have prospectively study Acute Physiology and Chronic Health Evaluation (APACHE) II prognostic system and Simplified Acute Physiology Score (SAPS) II in patients admitted in the adult ICU of All India Institute of Medical Sciences (AIIMS), New Delhi India. Firstly, we have sought to evaluate the ability of APACHE II and SAPS II scoring systems as a predictor of survival for patients admitted in ICU of AIIMS. Secondly, we have sought to compare the performance of the APACHE II and SAPS II scoring system in adult ICUs of AIIMS.

\section{INTRODUCTION}

India is a vast nation with varied forms of health care problems of a developing country. The major health problems include malnutrition and infections. Hence less importance has been given to the specialty of critical care and the need is only recently being recognized.

The history of critical care medicine in India started in the late 1960s and early 1970s with the development of coronary care units in Bombay and a few other large cities in India. The first coronary care unit in India was started in 1968 at Wing Edward Memorial Hospital in Bombay. Ventilator support was primitive and was generally offered as a terminal therapeutic approach. In the middle and later 1960s, the unit at the Breach candy hospital in Bombay started overall critical care with intensive respiratory care and successful support of critical illness due to a wide spectrum of diseases. Intensive care at the point in time lacked good monitoring facilities and was initially offered in a few designated rooms within a general ward.

After the mid 1980s, and particularly in 1990s, there has been a significant improvement in standards of care, particularly evident in the larger teaching and private hospitals in the cities of India. In 1993, some of younger physicians in Bombay engaged either in critical care medicine or acute medicine decided to further the cause of this specialty by forming the Indian Society of critical care medicine. Large teaching hospitals (e.g. AlIMS, Delhi and some large private hospitals in Bombay, Madras) have separate medical, surgical, pediatric coronary, cardio thoracic and neurological ICUs.

These are exceptions and most hospitals have all-purpose ICUs or at the most medical, surgical and coronary care unit. The other disturbing feature is that critical care is poor or nonexistent in district hospitals to which so many ill peoples from villages and smaller cities gravitate (Ahluwalia et al., 1999).

In country like India with meagre resources, there is an urgent need 
to identify variables, which have an implication on the prognosis. As most of scoring systems were developed in the west, they need to be validated in the Indian context. Only a few reports on the predictor of outcome in ICUs are available from India (Sackett et al., 1991).

\section{STUDY RATIONALE}

The demand for intensive care treatment is increasing and as such most of the ICUs are already overcrowded. Very often it is not possible to provide ICU care to all patients who require it. In such a setting it is very useful to be able to have a system of knowing which patient has a better chance of survival in the ICU. In a country like India with meager resources, this becomes all the more important. There is therefore an urgent need to be able to identify variables and scoring systems which have a direct implication on the prognosis.

As most of the prognostic scoring systems have been developed in the west, they need to be validated in the Indian context (Ahluwalia et al., 1999). Also as an Indian patient population and facilities are different, it is necessary to validate a prognostic scoring system, which will be applicable in an Indian context.

\section{MATERIALS AND METHODS}

The study was conducted prospectively in medical and surgica mechanically ventilated patients at an 8 bedded adult ICUs (medical and anesthesia) of AlIMS, a tertiary Institute in New Delhi, and was carried out over 7 months. A total of 125 subjects who were critically ill on mechanical ventilator and fulfilled the inclusion criteria were enrolled in the study by using convenience sampling technique. Data were collected through use of a standardized tool, APACHE II and SAPS II scoring system scale. A semi structured questionnaire was used for collecting information about demographic variables and general health conditions. The study was carried out after the approval of institute ethical committee. Informed consent was obtained from each respondent. Strict confidentiality of information was maintained. Data were summarized by using descriptive statistical methods e.g. mean and standard deviation was used for all continuous variables (demographic variables, physiological and biochemical variables). APACHE II day 1 , APACHE II day 2 , SAPS II day 1 and SAPS II day 2 points were assigned to all patients by calculating their individual score and predicted risk of mortality. Performance of the severity scoring system was assessed by Logistic regression analysis. The final outcome was labeled as alive or dead at the time of discharge. The calibration of the model was analyzed by calculating Standardized mortality ratio (SMR), whereas discrimination was evaluated by calculating area under a receiver operating characteristic (ROC) curve. All data were entered into the computer for statistical analysis with software SPSS version 12.

\section{RESULTS}

125 consecutive patients admitted in an 8 bedded adult ICUs were studied prospectively. The age of the patients ranged between 18 to 86 years (mean age 48.44 years). Males comprised $76(60.8 \%)$ and female comprised $49(39.2 \%)$ of the total number of patients. Of the 125 patients, $43(34.4 \%)$ died and 82 (65.6\%) survived. In demographic characteristics, age was significant $(p=0.001)$ in predicting outcome in mechanically ventilated patients. There were no relationship between outcome and gender, religion, education, occupation, family type, or personal habits such as alcohol intake and smoking.

Multivariate logistic regression was used to determine the relationship between the outcome and score of each scoring system on the day of admission, and after 24 hours of admission to the adult ICUS of AIIMS. The APACHE II and SAPS II score at admission and after 24 hours was also compared in the survivors and non-survivors.

The relationship between outcome and APACHE II score on the day of admission was statistically significant $(p=0.007)$. However, a greater statistically significant difference $(p=0.000)$ was found when APACHE II score was applied after 24 hours after admission. Likewise the relationship between outcome and SAPS II score on the day of admission and after 24 hours of admission was found to be statistically highly significant $(p=0.000)$.

Multiple logistic regression describes the relationship between a dependent variable and more than one independent variable. Multiple logistic regression has also been referred to as the log-odds method i.e. the odds ratio (OR) (Sackett et al., 1991). In analyzing the results of odds ratio for each scoring system (see Table 1) by using multivariate logistic regression analysis, it was found that all the scoring systems were predicting outcome well in mechanically ventilated patients admitted in the adult ICUs of AlIMS. For APACHE II day 1 scoring system $(O R=2.385 ; p=0.026)$ was used to predict outcome 2 times. The APACHE II day 2 scoring system $(\mathrm{OR}=$ 2.924; $p=0.007$ ) was also used to predict outcome twice. Both the scoring systems were statistically significant and predicted outcomes independently. However, APACHE II day 2 scoring system was more statistically robust than APACHE II day 1 . Likewise for the SAPS II day 1 and day 2 scoring systems $(O R=4.45 ; p=0.000$ and $O R$ $=6.243 ; p=0.000)$, SAPS $\|$ day 1 and day 2 were both able to predict outcomes independently at a statistically significant level. Among all the scoring system, SAPS II day 2 scoring system was highly predictive at identifying outcomes in mechanically ventilated patients.

The performance of the model was evaluated using discrimination and calibration. Accuracy of discrimination was assessed by the area under the receiver operating characteristic (ROC) curve. ROC curve was used to compare the performance of APACHE II day 1 and 2 scoring systems with SAPS II day 1 and 2 scoring systems.

By using ROC curve (see Figures 1-4 and Table 2), the area under curve for the APACHE II day1, APACHE II day 2, SAPS II day1 and day 2 were $0.648,0.740,0.744$ and 0.802 respectively. This means that the APACHE II day1 and 2, SAPS II day1 and day 2 scoring systems are predicting outcomes by $64.8 \%, 74 \%, 74.4 \%$ and $80.2 \%$ respectively and all are statistically significant. SAPS II day 2 scoring system was found to be the best predictive amongst all.

Table 1. Logistic model for the APACHE II D1, APACHE II D2, SAPS II D1 and SAPS I| D2 severity scoring systems for predicting outcome (D1 = day $1, \mathrm{D2}=$ day 2 )

\begin{tabular}{|c|c|c|c|c|}
\hline Outcome & Odds Ratio & Significance & \multicolumn{2}{|c|}{$\mathbf{9 5 \%}$ Cl for OR } \\
\cline { 4 - 5 } & & & Lower & Upper \\
\hline APACHE II D1 & 2.385 & 0.026 & 1.111 & 5.120 \\
\hline APACHE II D2 & 2.924 & 0.007 & 1.348 & 6.345 \\
\hline SAPS II D1 & 4.451 & 0.000 & 2.010 & 9.855 \\
\hline SAPS II D2 & 6.243 & 0.000 & 2.753 & 14.157 \\
\hline
\end{tabular}

Table 2. Comparison of different models for outcome prediction (D1 = day 1, D2 = day 2)

\begin{tabular}{|c|c|c|c|c|}
\hline & $\begin{array}{c}\text { APACHE } \\
\text { II D1 }\end{array}$ & $\begin{array}{c}\text { APACHE } \\
\text { II D2 }\end{array}$ & $\begin{array}{c}\text { SAPS II } \\
\text { D1 }\end{array}$ & SAPS II D2 \\
\hline Cut off point & 22.50 & 20.50 & 48.50 & 44.50 \\
\hline Area under ROC curve & 0.648 & 0.740 & 0.744 & 0.802 \\
\hline ROC curve & 0.648 & 0.740 & 0.744 & 0.802 \\
\hline Significance p & 0.007 & 0.000 & 0.000 & 0.000 \\
\hline Sensitivity & $65.1 \%$ & $67.4 \%$ & $69.8 \%$ & $72.1 \%$ \\
\hline Specificity & $43.9 \%$ & $41.5 \%$ & $34.1 \%$ & $29.3 \%$ \\
\hline
\end{tabular}


Table 3. Predicted mortality and standardized mortality ratio

\begin{tabular}{|c|c|c|c|c|}
\hline & $\begin{array}{c}\text { APACHE } \\
\text { II Day 1 } \\
\text { Scoring } \\
\text { system }\end{array}$ & $\begin{array}{c}\text { APACHE } \\
\text { II Day 2 } \\
\text { Scoring } \\
\text { system }\end{array}$ & $\begin{array}{c}\text { SAPS II Day } \\
\text { 1 Scoring } \\
\text { system }\end{array}$ & $\begin{array}{c}\text { SAPS II Day } \\
\text { 2 Scoring } \\
\text { system }\end{array}$ \\
\hline $\begin{array}{c}\text { Mean score } \\
\pm \text { SD }\end{array}$ & $22.89 \pm 6.77$ & $20.85 \pm 6.88$ & $47.85 \pm 15.17$ & $42.66 \pm 14.10$ \\
\hline $\begin{array}{c}\text { Observed } \\
\text { mortality }\end{array}$ & $34.4 \%$ & $34.4 \%$ & $34.4 \%$ & $34.4 \%$ \\
\hline $\begin{array}{c}\text { Predicted } \\
\text { mortality }\end{array}$ & $52.27 \%$ & $44.06 \%$ & $42.32 \%$ & $35.52 \%$ \\
\hline $\begin{array}{c}\text { Standardized } \\
\text { mortality ratio }\end{array}$ & 0.65 & 0.77 & 0.81 & 0.96 \\
\hline
\end{tabular}

Table 4. Predicted risk of death in survivors and non-survivors $(P D R=$ predicted death rate)

\begin{tabular}{|c|c|c|c|c|}
\hline Outcome & $\begin{array}{c}\text { APACHE II } \\
\text { PDR day 1 }\end{array}$ & $\begin{array}{c}\text { APACHE II } \\
\text { PDR day 2 }\end{array}$ & $\begin{array}{c}\text { SAPS II } \\
\text { PDR day 1 }\end{array}$ & $\begin{array}{c}\text { SAPS II } \\
\text { PDR day 2 }\end{array}$ \\
\hline $\begin{array}{c}\text { Survivors } \\
\mathrm{n}=82\end{array}$ & $41.96 \pm 19.87$ & $34.25 \pm 18.88$ & $34.25 \pm 23.87$ & $24.67 \pm 18.88$ \\
\hline $\begin{array}{c}\text { Non-survivors } \\
\mathrm{n}=43\end{array}$ & $53.70 \pm 20.87$ & $51.37 \pm 19.75$ & $56.25 \pm 24.91$ & $50.64 \pm 23.88$ \\
\hline
\end{tabular}

ROC Curve

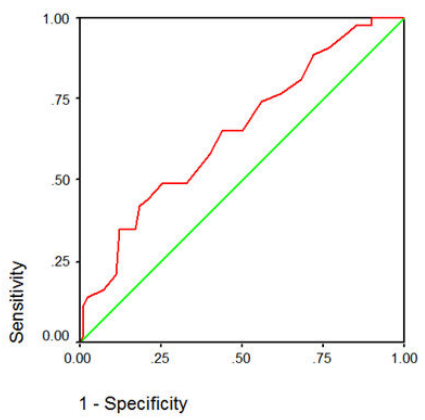

Figure 1. APACHE II Day1 Scoring System $(\mathrm{AUC}=\mathbf{0 . 6 4 8})$

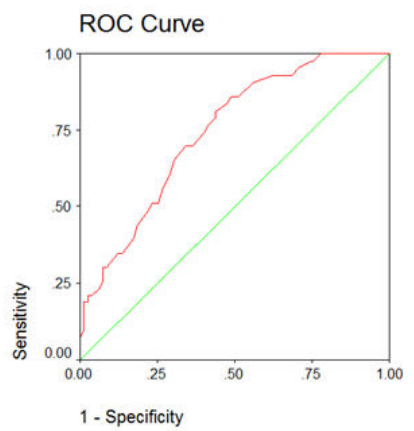

Figure 3. SAPS II Day 1 Scoring System $(A \cup C=0.744)$

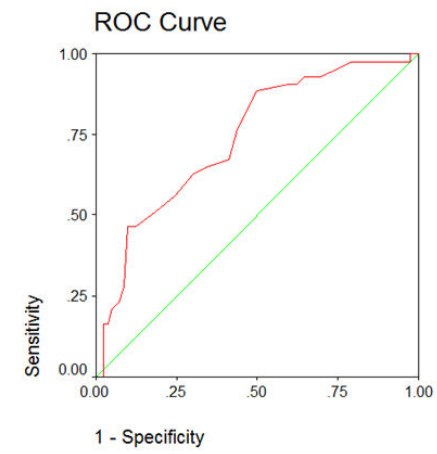

Figure 2. APACHE II Day 2 Scoring System System $(A \cup C=0.740)$

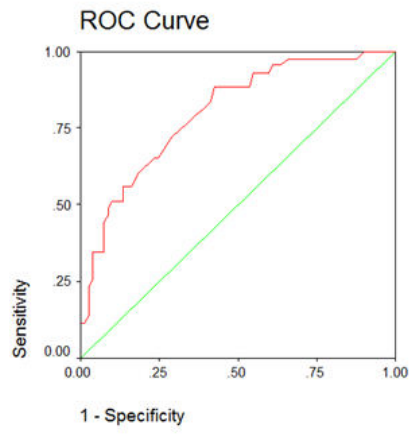

Figure 4. SAPS II Day 2 Scoring System (AUC $=0.802$ )
The other aspect of the validation process that was used was calibration. Calibration involves the comparison of the true outcomes to the model-estimated outcomes over the entire range of risk (Berwick \& Thibodeau, 1983). The most commonly used goodnessof-fit test is the standardized mortality ratio (SMR) (Lemeshow \& Hosmer, 1982), (Hosmer \& Lemeshow, 1980). SMR is a ratio of observed mortality divided by predicted mortality. Calibration of the different models is shown in Table 3. Calibration for APACHE II day 2 scoring system $(\mathrm{SMR}=0.77$ ) was good as compared to APACHE II day1scoring system $(S M R=0.65)$. The calibration was better than APACHE II day 1 and day 2 scoring system for the SAPS II day 1 scoring system $(S M R=0.81)$. The calibration was best for the SAPS II day 2 scoring system $(\mathrm{SMR}=0.96)$.

On analysis of the above data and comparing odds ratio, discrimination i.e. area under ROC curve and calibration (SMR), it was found that for prediction of outcome of patients admitted to adult ICUs of AIIMS, APACHE II day 2 scoring system was better than APACHE II day 1 scoring system. The SAPS II day 1 scoring system was even better than other scoring systems however the SAPS II day 2 scoring system's predictive value was even better among the other scoring systems. The survivors had a significantly lower predicted risk of death than non-survivors in all the illness severity scoring systems (see Table 4).

\section{DISCUSSION}

Many scoring systems have been formulated to predict the outcome of critically ill patients in the last two decades. These includes APACHE I (Knaus et al., 1981), APACHE II (Knaus et al., 1985a), APACHE III (Knaus et al., 1991), TISS (therapeutic intervention scoring system) (Cullen et al., 1974), SAPS (simplified acute physiology score): SAPS I (Le Gall et al., 1984) and SAPS II (Le Gall et al., 1993), MPM (mortality prediction model): MPM I (Lemeshow et al., 1985) and MPM II (Lemeshow et al., 1999) OSF (organ system failure) (Knaus et al., 1985b) and ODIN (organ dysfunction and infection) (Fagon et al., 1996) scores.

All of these scoring systems have been formulated in the developed world, where the patient population and resources are different. The applicability of these scoring systems needs to be validated in an Indian population as different ICUs have a varying spectrum of diseases and the severity of the diseases is also not the same. With sophisticated and expensive technologies becoming increasingly available in India, there is a need to develop or adapt scoring systems that are valid for Indian population. Only a few reports on the predictors of outcome in the intensive care units are available from India. Predictive models must demonstrate ease and speed of use, general applicability and accuracy, before they can become clinically useful. The APACHE II and SAPS II score have already been used extensively in multidisciplinary ICUs on thousands of patients. It measures the degree of physiologic derangement from the acute illness, premorbid chronic conditions, and the patient's age - factors which are known to influence survival.

In present study, APACHE II scoring system and SAPS II scoring system were studied in patients admitted to the adult ICUs, similar to how they have been extensively studied in the western population.

The present study was prospectively conducted with 125 patients admitted in the adult ICUs of AIIMS. It was found that SAPS II day 2 scoring system had a better predictive value $(p=0.000)$ than APACHE II day 1 , APACHE II day 2 and SAPS II day 1 scoring system $(p=$ $0.026,0.007 \& 0.000$ ). By comparing area under ROC curve, it was found that the SAPS II day 2 scoring system (area under ROC curve 0.802 ) was better than SAPS II day 1 scoring system (area under ROC curve 0.744), APACHE II day 1 scoring system (area under ROC curve 0.648) and APACHE II day 2 scoring system (area under ROC curve 0.740 ) in predicting outcome in ICU patients.

A similar study was conducted in a multidisciplinary ICU of teaching hospital, a single centre population in Italy (Capuzzoet al., 2000), where they studied 1721 consecutively admitted patients. The area under the ROC curve was higher than 0.8 for the SAPS II scoring system. This supports the present study. Another similar study was 
conducted in a medical ICU in Australia, where they studied 242 patients and SAPS II yielded a significantly superior discrimination between survivors and non survivors. The areas under ROC curve were 0.776 for APACHE II and 0.825 for SAPS II scoring system (Schellongowski et al., 2004).

In the present study, the results show an acceptable discrimination for all scoring systems in a population of critically ill mechanically ventilated patients with a $p$ value of $<0.05$ for all the scoring system (models). The discrimination between survivors and non-survivors appeared to be superior for SAPS II compared to other scores. The APACHE II day 1 , day 2 and SAPS II day 1 could not be superior to the SAPS II day 2 severity-of-illness scores, the area under ROC curve was $0.648,0.740,0.744$ in the present study population (moderate) compared with an area under curve of 0.806 in the original publication. The area under curve of SAPS II was 0.802 , which can be regarded as a satisfactory good value (Schellongowski et al., 2004). Prior studies concluded that the accuracy of the illness severity scoring systems were similar for patients with similar SAPS II day 1 score in the United States and other developed countries. This may be due to the fact that intensive care in India is less expensive compare to other facilities, and adequate manpower is available as compared to the west. In addition, the SAPS II scores may underestimate the mortality for Indian patients because of differences in case mix, higher lead time between onset of admission and treatment before ICU admission, and possible inappropriateness of age points derived from developed countries patients for Indian subjects because of a higher burden of disease at lower ages in Indian patients.

In this study, calibration was assessed for the various scoring systems by comparing standardized mortality ratio (SMR). Standardized mortality ratio (SMR) is a ratio of observed mortality divided by predicted mortality for a given scoring system. SMR for APACHE II day 1 scoring system was 0.65 , for APACHE II day 2 scoring system was 0.77 , for the SAPS II day 1 scoring system was 0.81 and for SAPS II day 2 scoring system was 0.96 . Thus, the results demonstrate that progression from APACHE II day 1 scoring system to the SAPS II day 2 scoring system results in a progressively improved SMR (which approaches 1(0.96)).

In a similar study (Capuzzo et al., 2000), considered calibration, and the SMR demonstrated that the hospital mortality observed in the study (SMR=0.98) patients did not differ significantly from that predicted by SAPS II models/scoring system (SMR=0.96). Another similar study from Germany (Markgraf et al., 2000), considering calibration, with SMR $=0.88$ for their patients for SAPS II scoring system. It was clearly found that calibration was even better with a close fit for SAPS II day 1 and day 2 scoring system only. Therefore, data from the present study more closely resembles those of SAPS II day 2 data base, which demonstrates a higher degree of overall goodness of fit of SAPS II day 2 compared to APACHE II day 1 , day 2 and SAPS II day 1 . Although discrimination and calibration were significantly better for all the scoring systems, discrimination and calibration was best with a close fit for SAPS II day 2 only. This shows that mortality of Indian patients' population was 0.96 times that for patients with similar SAPS II scores in ICUs in the developed countries. This may be due to the fact that better facilities exist at AlIMS as it is an apex institution. AlIMS adult ICUs have most of the modern facilities including mechanical ventilators, ABG machines, bedside monitors, and the availability of round the clock nurses and doctors. This may be the reason that observed mortality in our ICU $(34.4 \%)$ was comparable to the predicted mortality (35.52\%).

Thus from above statistical analysis, it seems that APACHE II day 2 scoring system is better in predicting outcome in ICU patients than APACHE II score at admission (Day 1); and the SAPS II scoring system was the best predictor of outcome overall in an adult ICUs of AlIMS.
The suggested prediction rule is based on a limited number of observation in a general purpose medical ICU, where only one thirds of patients required ventilatory assistance. The 8-bedded anesthesia and medical ICU, however, has facilities for ventilating eight-eight patients at a given time with modern ventilators, and noninvasive ventilators are also available. Modern ABG machine is available which besides assessing arterial blood gases, also measures electrolytes, serum osmolality, hematocrit and serum lactate levels. Adequate bedside monitoring and other intensive care facilities are also available for all the patients. One junior resident, one senior resident and 1:1 ratio of nurses for each bed are available round the clock. The findings from this study may not be generalized to other ICUs with different spectrum of patients and available facilities. However, it is also important to appreciate the moral dilemma we as health care provider will have to face in the usage of these scoring systems, namely to use empirically devised prognostic estimates in to a larger decision making framework of allocating a bed to a critically ill patient in the ICU.

Additionally, the SAPS II scoring system day 2 in the present study needs to be validated at other centers in India. This study should stimulate further research in this field so as to develop/validate various scoring systems, which can accurately predict outcome in ICU patients in the Indian context.

\section{IMPLICATIONS}

Scoring system helps in effective planning of care to meet the needs of critically ill patients and their families who have complex, multi system problems. Predictability is one of the prime roles of severity scoring systems, one to expect a certain trajectory of illness. Admission and discharge decisions in the ICU can be done on the basis of this severity scoring system. Subjects who have been diagnosed with higher predicted mortality rates needs more vigilant monitoring and highly specialized nursing \& medical care. Optimized distribution of medical, nursing and financial recourses is of crucial importance in the delivery of health care, particularly in the poor developing countries of the world. To this end critical care physicians and nurses often have to make decisions as to which patients are likely to derive the maximum benefit from admission to a critical care unit. Therefore, patients with chronic terminal illnesses, with the end close at hand, should be given every care at home or in the ward of a hospital, but not in critical care units and this can be only achieved by using severity scoring system.

\section{CONCLUSION}

The results of this study demonstrated that common ICU illness severity scoring systems (APACHE II day 1 , APACHE II day 2 , SAPS II day 1 and SAPS II day 2 ) were of acceptable value to describe severity of illness and to estimate outcome in a group of critically ill mechanically ventilated patients in an Indian ICU setting. Among the systems, the Simplified Acute Physiology Score (SAPS II) day 2 illness severity scoring system had greater predictive value. The results of this study add value and can aid nurses, clinicians and relatives of patients on mechanical ventilation in deciding on the probable outcome and management decisions.

\section{Acknowledgement}

The author expresses heart felt gratitude to Dr. Chandra Lekha, Professor and Head, Department of Anesthesia \& Critical care, AlIMS and Dr. Manju Vatsa, Principal, College of Nursing, AlIMS for permitting me to do the study and for her constructive criticisms and constant support, to Dr. R. M. Pandey, professor \& Head, and Mr. Alok Dwiwedi, Department of Bio Statistics for their statistical assistance. We express our thanks to all staff of anesthesia \& Critical care and medicine ICU for their kindness and cooperation. 


\section{REFERENCES}

Ahluwalia G, Pande JN, Sharma SK (1999). Prognostic Scoring for critically ill hospitalized patients. Indian Journal of Chest Diseases and Allied Science 41, 201-206.

Berwick DM, Thibodeau LA (1983). Receiver operating characteristic analysis of diagnostic skill. Medical Care 21, 87685.

Capuzzo M, Valpondi V, Sgarbi A et al. (2000). Validation of severity scoring systems SAPS II and APACHE II in a single-center population. Intensive Care Medicine 26(12), 1779-85.

Cullen DJ, Civetta JM, Briggs BS (1974). Therapeutic Intervention Scoring System; A method for quantitative patient care. Critical Care Medicine 2, 57-60.

Fagon JY, Chastre J, Novara A, Medioni P, Gibert C (1996). Characterization of intensive care unit patients using ODIN model. Intensive Care Medicine 22, 564-70.

Hanley JA, McNeil BJ (1982). The meaning and use of the area under a receiver-operating characteristic (ROC) curve. Radiology 143, 29-36.

Hosmer DW, Lemeshow S (1980). A goodness-of-fit test for the multiple logistic regression model. Communications In Statistics A10, 1043-69.

Knaus WA, Zimmerman JE, Wagner DP, Draper EA, Lawrence DE (1981). APACHE - acute physiology and chronic health evaluation: a physiologically based classification system. Critical Care Medicine 9, 591-7.

Knaus WA, Draper EA, Wagner DP, Zimmerman JE (1985a). APACHE II: A severity of disease classification system. Critical Care Medicine 13, 818-29.

Knaus WA, Draper EA, Wagner DP, Zimmerman JE (1985b). A prognosis in acute organ system failure. Annals of Surgery 202, 685-93.

Knaus WA, Wagner DP, Draper EA (1991). The APACHE III prognostic system : Risk prediction of hospital mortality for critically ill hospitalized adults. Chest 101, 1619-36.

Le Gall JR, Loirat P, Alperovitch A, Glaser P, Granthil C, Mathieu D (1984). A Simplified Acute Physiology Score for ICU patients. Critical Care Medicine 12, 975-7.
Le Gall JR, Lemeshow S, Saulnier F (1993). A new Simplified Acute Physiology Score (SAPS II) based on a European / North American multicenter study. JAMA 270, 2957-63.

Lemeshow S, Hosmer DW (1982). A review of goodness of fit statistics for use in the development of logistic regression models. American Journal of Epidemiology 115, 91-106.

Lemeshow S, Teres D, Pastides H, Spitz-Avrunin J, Steingrub JS (1985). A method for predicting survival and mortality of ICU patients using objectively derived weights. Critical Care Medicine 13, 519-25.

Lemeshow S, Teres D, Klar J, Spitz- Avrunin J, Gehlbach SH, Rapoport J (1999). Mortality Probability Models (MPM II) based on an international cohort of intensive care unit patients. JAMA 270, 2478-86.

Markgraf R, Deutschinoff G, Pientka L, Scholten T (2000). Comparison of acute physiology and chronic health evaluations II and III and simplified acute physiology score II: a prospective cohort study evaluating these methods to predict outcome in a German interdisciplinary intensive care unit. Critical Care Medicine 28(1), 26-33.

Meyer A, Hunt JP (1997). Predicting survival in the intensive care unit. Current Problems in Surgery, 34(7): 527-99.

Sackett DL, Hayn RB, Guyatt GH, Tugwell P (1991). Clinical Epidemiology. Boston: Little, Brown and Co.

Schellongowski P, Benesch M, Lang T et al. (2004). Comparison of three severity scores for critically ill cancer patients. Intensive Care Medicine 30(3), 430-6.

Schreiderman LJ, Jecker NS, Jonsen AR (1990). Medical Futility; its meaning and ethical implications. Annals of Internal Medicine 112, 948-54.

Udwadia FE (1995). An Introduction to Critical Care. Delhi: Oxford University Press.

Zweig MH, Campbell G (1993). Receiver-operating characteristic (ROC) plots. A fundamental evaluation tool in clinical medicine. Clinical Chemistry 39(4), 561-77. 